\title{
Interactions Between Acanthamoeba culbertsoni and Pathogenic Bacteria and their Inhibition by Lectin- Antibodies
}

\author{
Suk-Yul Jung \\ Department of Biomedical Laboratory Science, Molecular Diagnostics Research Institute, Namseoul University, \\ Cheonan 31020, Republic of Korea.
}

\begin{abstract}
In this study, using pathogenic and non-pathogenic bacteria, it was analyzed whether a polyclonal serum and a monoclonal antibody to $A$. culbertsoni mannose-binding protein (MBP) could inhibit its interaction. The association of the amoeba with E. coli 0157: $\mathrm{H7}$ was very strong at a level of over $100 \%$, but the non-pathogenic $E$. coli strain was about five times lower at $22 \%$. Pathogenic $K$. pnueumoniae also showed high association with amoeba by about $92 \%$ as compared with pathogenic $E$. coli $0157: \mathrm{H} 7$ and S. agalactiae. The polyclonal serum to MBP inhibited $E$. coli 0157:H7 association to amoeba 2.5 times more than untreated E. coli 0157:H7. Monoclonal antibody to MBP also inhibited bacterial association with amoeba but was not stronger than the polyclonal serum. Pathogenic $E$. coli 0157:H7 showed about $88 \%$ invasion into amoeba and decreased about $22 \%$ as compared with associated E. coli 0157:H7. Polyclonal serum to MBP inhibited about 55\%, 50\%, and 44\% in E. coli 0157:H7, $K$. pneumoniae and $S$. agalactiae, respectively. The invasion of $K$. pneumoniae and $S$. agalactiae was not high as polyclonal serum but was about $8 \%$ to $10 \%$ weaker than polyclonal serum. The pathogenic strains of $K$. pneumoniae and $S$. agalactiae showed less decrease in survival as shown at invasion than E. coli 0157:H7 without antibody. This study provided the information that the pathogenic bacteria could be more interactive with $A$. culbertsoni trophozoites as a reservoir host than non-pathogenic $E$. coli, and the amoeba should interact with bacteria by the MBP lectin.
\end{abstract}

Keywords: Acanthamoeba culbertsoni, Association, Bacteria, Lectin, Invasion

*Correspondence: syjung@nsu.ac.kr; +82-041-580-2723

(Received: March 28, 2020; accepted: July 02, 2020)

Citation: Jung S-Y. Interactions Between Acanthamoeba culbertsoni and Pathogenic Bacteria and their Inhibition by LectinAntibodies. J Pure Appl Microbiol. 2020;14(3):1687-1693. doi: 10.22207/JPAM.14.3.07

(c) The Author(s) 2020. Open Access. This article is distributed under the terms of the Creative Commons Attribution 4.0 International License which permits unrestricted use, sharing, distribution, and reproduction in any medium, provided you give appropriate credit to the original author(s) and the source, provide a link to the Creative Commons license, and indicate if changes were made. 


\section{INTRODUCTION}

Acanthamoeba is a free-living protozoa isolated in a variety of environments, including air, soil, tap water and swimming pools. It is known to be one of the most ubiquitous protozoans $\mathrm{s}^{1-3}$. The ability of Acanthamoeba to survive in such diverse environments is evidence that it can interact with bacteria, eat and live. Acanthamoeba acts as a reservoir for several pathogenic bacteria such as Escherichia coli $\mathrm{K} 1$ as an agent of meningitis ${ }^{4}$, Legionella pneumophila as an agent of Legionnaire's disease ${ }^{5}$, Pseudomonas aeruginosa as an agent of keratitis ${ }^{6}$, etc.

Environmental bacteria commonly colonize and form cluster such as biofilms, which generally contain a number of prokaryotic and eukaryotic cells associating with adhesion molecules and secreted compounds ${ }^{7}$. In addition, because the process of infection between amoebae and macrophages is very similar, amoeba is a powerful model for studying bacteriamacrophage interactions ${ }^{8,9}$. Their interactions were inferred from the phagocytic ability of Acanthamoeba and macrophage. Bacteria contain a polysaccharide layer, e.g., a capsule outside of the bacterial wall ${ }^{10}$. In a previous study, a polysaccharide glucuronoxylomannan composed of a capsule of Cryptococcus neoformans was ingested by $A$. castellanii, indicating an additional similarity between the mechanisms by which amoeba and phagocytes interact with cryptococcal components ${ }^{11}$. Moreover, receptors in Acanthamoeba are of interest in the interactions between amoeba and bacteria. One of those, mannose-binding protein (MBP) of $A$. castellanii and $A$. culbertsoni, was known to play an important role in the pathogenesis of infection by mediating the attachment of parasites to the host cells ${ }^{12,13}$. It may mediate the interaction of amoeba with other cells such as bacteria. In this study, using pathogenic and non-pathogenic bacteria such as E. coli 0157:H7, K. pnuemoniae, S. agalactiae and E. coli $\mathrm{DH} 5 \alpha$, it was analyzed whether a polyclonal serum and a monoclonal antibody to MBP of $A$. culbertsoni could inhibit its interactions, such as bacterial association, invasion and survival. Moreover, the amount of difference between interactions were analyzed as compared with interaction untreated with antiserum or antibody.

\section{MATERIALS AND METHODS \\ Amoeba culture}

A. culbertsoni trophozoites (ATCC No. $30,171)^{14,15}$ were cultured without shaking in $12 \mathrm{ml}$ of PYG medium $(0.75 \%$ proteose peptone (Kisan Bio, Seoul, Korea), $0.75 \%$ yeast extract (Kisan Bio, Seoul, Korea) and g1.5\% glucose (Sigma-Aldrich Co., St. Louis, MO, USA)) in a T75 culture flask at $37^{\circ} \mathrm{C}$. The media was refreshed $17-20$ hours prior to experiments ${ }^{16,17}$. The result was more than $99 \%$ amoebae in the trophozoite form, which was subsequently used for carbohydrate selection.

\section{Bacterial strains}

Three harmful bacteria, e.g., E. coli 0157:H7 (ATCC No. 43895), K. pnuemoniae (ATCC No. BAA-1705) and S. agalactiae (ATCC No. 13813), one non-pathogenic E. coli DH5 $\alpha$ (KCTC No. 22002) were applied for adhesion and invasion assay. $E$. coli strains and $K$. pneumoniae are rod-shaped but S. agalactiae exhibits a round form. The analysis of the two assays was also performed according to the different sized-bacteria. In this study, single colonies were subcultured into other tryptic soy agar (TSA, MB cell, Korea) plates at $37^{\circ} \mathrm{C}$, and were checked by Gram-staining procedures ${ }^{18}$.

MBP purification and production of its antibodies

The protocols to purify MBP were described in the previous report13. Briefly, trophozoites of A. culbertsoni cultured in a T75 flask were washed three times with phosphate buffered saline (PBS) (Sigma-Aldrich Co., St. Louis, MO, USA), then centrifuged and dissolved with a lysis buffer containing with $50 \mathrm{mM}$ Tris- $\mathrm{HCl}, 50 \mathrm{mM} \mathrm{CaCl}, 150 \mathrm{mM} \mathrm{NaCl}, 1 \mathrm{mM}$ phenyl methanesulfonyl fluoride (PMSF), $2 \mathrm{mM}$ $\beta$-mercapto ethanol, 0.5\% CHAPS (Sigma-Aldrich Co., St. Louis, MO, USA)) by a sonicator for 2 min with $20 \mathrm{~W}$. The total amoebial lysate was collected by centrifugation $\left(13,000 \mathrm{rpm}, 1 \mathrm{~h}, 4^{\circ} \mathrm{C}\right)$ and purified with $\alpha$-D-mannose agarose (SigmaAldrich Co., St. Louis, Missouri, USA) affinity column (Qiagen, CA, USA). Unbound lysate was removed with the washing buffer and the bound lysate were eluted with $150 \mathrm{mM}$ mannose ${ }^{12,19,20}$. The eluates were biweekly immunized into BALB/C mice (Narabiotech, Pyeongtaek, South Korea) three times for six weeks, and then polyclonal serum was isolated. The monoclonal antibody, the selected hybridomas clones kindly provided 
from the previous study ${ }^{13}$, were moved into a T75 culture flask. The monoclonal antibody was a IgM class of kappa chain named DG11.

Assay of amoeba-bacteria interactions Acanthamoeba can ingest bacteria such as $E$. coli, S. aureus, Pseudomanas aeruginosa, etc. as a reservoir host. Prior to ingestion in the cytoplasm of Acanthamoeba, bacteria can adhere to the outer membrane of the amoeba and then enter its cytoplasm. The processes of bacterial adherence and entrance were called association and invasion, respectively. Their experimental procedures were referred by Jung et al., $2007^{3}$ and modified a little. Briefly, A. culbertsoni trophozoites were cultured in 24-well culture plates with PYG medium. Acanthamoeba was incubated with each bacterium $\left(2 \times 10^{5} \mathrm{cfu} / 0.5 \mathrm{ml}\right.$ of PBS) and plates incubated for $1 \mathrm{~h}$ at room temperature (RT). Obtained single colonies were diluted with $0.85 \% \mathrm{NaCl}$ and were set to 0.5 of McFaland turbidity producing $1.5 \times 10^{3}$ to $1.5 \times 10^{6}$ colony forming units (CFU) $/ \mathrm{ml}^{21}$. After the incubation, amoebae were washed 3 times with PBS to remove unassociated bacteria, and amoebae were counted using a haemocytometer. Finally, amoebae were dissolved by adding SDS
(0.5\% final concentration) to each well for $30 \mathrm{mi}$ and the number of bacteria was enumerated by plating on nutrient agar plates ${ }^{4}$. The percentage of bacterial association was calculated as follows: recovered bacteria $(\mathrm{cfu}) /$ total bacteria $(\mathrm{cfu}) \times 100$ $=\%$ bacteria associated with Acanthamoeba.

Invasion assay represented entrance of bacteria into the cytoplasm of $A$. culbertsoni trophozoites. For the invasion assay, after the bacteria were incubated with amoebae, gentamicin antibiotics was added for $45 \mathrm{~min}$ (100 $\mu \mathrm{g} / \mathrm{ml}$, final concentration) to remove extracellular bacteria. Finally, amoebae were counted and the intracellular bacteria were also counted. The percentage of bacterial invasion was calculated as follows: recovered bacteria (cfu)/total bacteria (cfu) $\times 100=$ percentage of intracellular bacteria. For these association and invasion assays, the polyclonal serum or monoclonal antibody of DG11 to MBP was preincubated with $A$. culbertsoni trophozoites for 30 min prior to the assays and then incubated with bacteria. Invaded bacteria can survive within the amoeba cytoplasm and vacuoles. The invaded bacteria treated with gentamicin were allowed to survive within the

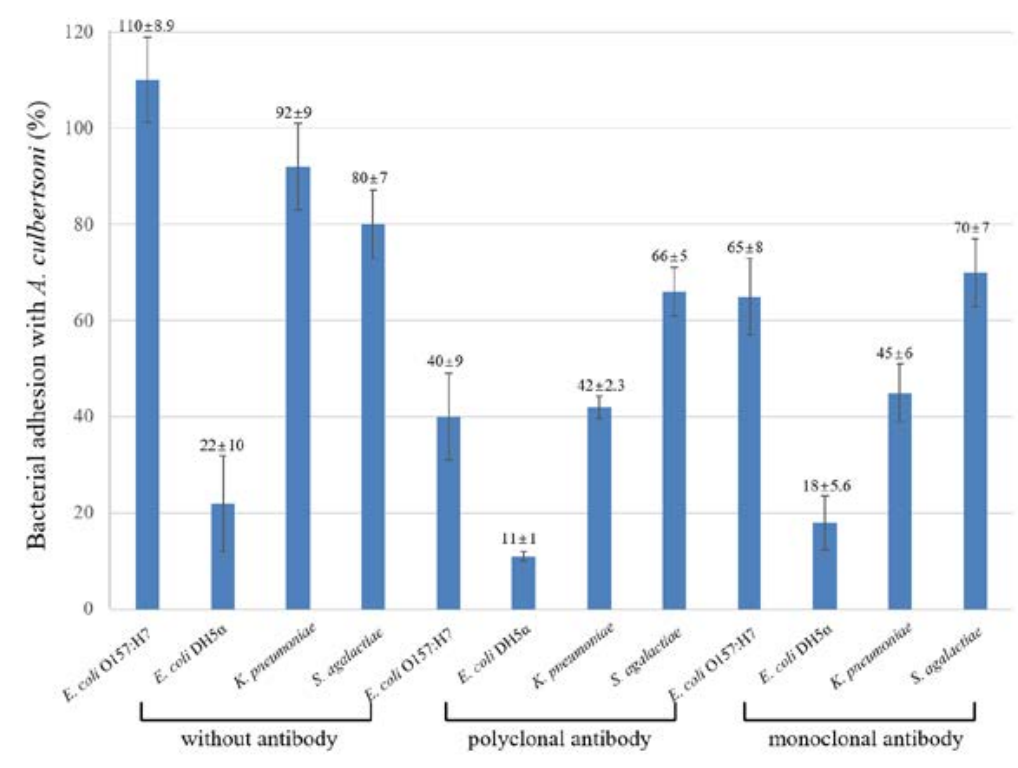

Fig. 1. Percentage of bacterial association with A. culbertsoni trophozoites. Pathogenic E. coli $0157: \mathrm{H7}, K$. pnuemoniae, S. agalactiae and one non-pathogenic $E$. coli $\mathrm{DH} 5 \alpha$ were analyzed by association, and antibodies to MBP was preincubated with the amoeba for $30 \mathrm{~min}$ prior to the association assay. This experiment was performed in triplicate wells with three times, and data were described by mean \pm standard deviation (SD) value. 
amoeba cytoplasm for 1 hour, and the surviving bacteria were calculated as mentioned above.

\section{Statistical analysis}

Statistical differences between groups or samples were determined by using a Student two-sample $t$ test. The difference was considered significant when $P$ was $<0.05$.

\section{RESULTS}

\section{Analysis of bacterial association with amoeba trophozoites}

To understand how many bacteria could adhere to $A$. culbertsoni trophozoites, an association assay was performed. A. culbertsoni trophozoites can possess or form a lot of pseudopodia which can associate with bacteria. Also, bacteria have various factors that associate with A. culbertsoni, e.g., OmpA, LPS, etc. $\mathrm{MBP}^{3}$. $\mathrm{MBP}$ in $A$. culbertsoni trophozoites were one of the association factors ${ }^{13}$, implying that antibodies would inhibit their association. The association of $E$. coli $0157: \mathrm{H} 7$ with amoeba was extremely strong at over $100 \%$, but non-pathogenic $E$. coli strain was about five times lower of $22 \%$ (Fig. 1). Another pathogenic strain, K. pnueumoniae, also showed high association with amoeba by about $92 \%$ as compared with pathogenic $E$. coli 0157:H7. S. agalactiae showing a round form of chains also had a high association with amoeba by about $80 \%$. Taken together, it suggested that pathogenic strains of bacteria could associate with $A$. culbertsoni trophozoites four or five times higher than non-pathogenic $E$. coli.

Effect of MBP antibodies for amoeba interactions with bacteria

The polyclonal serum to MBP inhibited E. coli 0157: $\mathrm{H} 7$ association to amoeba 2.5 times more than untreated E. coli O157:H7. The other three bacteria also inhibited their association with amoeba irrespective of bacterial pathogenicity. On the other hand, monoclonal antibody to MBP also inhibited bacterial association with amoeba as compared with antibody-untreated amoeba but not stronger than the polyclonal serum. Because of the polyclonal serum more antibodies recognized various epitopes, and the monoclonal antibody to only MBP would not show strong inhibition as compared with the polyclonal serum.

\section{Bacterial invasion by the MBP antibodies}

Amoebae have the potential to ingest the associated or adhered bacteria because bacteria are small-sized, and amoebae express pseudopodia

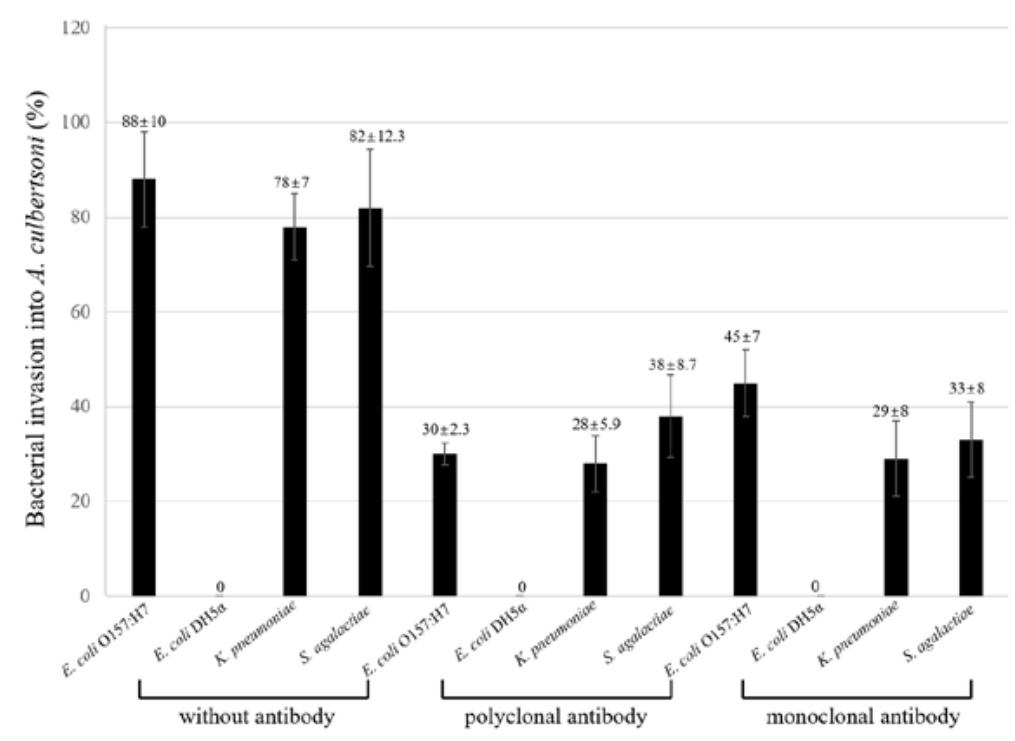

Fig. 2. Percentage of bacterial invasion into $A$. culbertsoni trophozoites. Pathogenic $E$. coli O157:H7, K. pnuemoniae, S. agalactiae and one non-pathogenic $E$. coli $\mathrm{DH} 5 \alpha$ were analyzed by invasion. Invasion assay was done post to the association assay above. This experiment was performed in triplicate wells with three times, and data were described by mean \pm standard deviation (SD) value. 
to eat bacteria ${ }^{1}$. Here, it was analyzed how much of a percentage of bacteria could invade the amoeba and also the polyclonal serum and monoclonal antibody above inhibited bacterial invasion by the treatment with the antibiotic gentamicin which could kill uninvaded remaining bacteria (Fig. 2). Bacterial invasion showed very similar patterns as the association results in Fig. 1, but the number of bacteria invading decreased. Pathogenic $E$. coli 0157:H7 showed about $88 \%$ of invasion into amoeba and decreased about $22 \%$ as compared with associated $E$. coli 0157:H7. On the other hand, associated non-pathogenic E. coli $\mathrm{DH} 5 \alpha$ with amoeba was about $22 \%$ but never invaded into amoebae. Polyclonal serum to MBP inhibited about 55\%, 50\%, and 44\% in E. coli O157:H7, $K$. pneumoniae and $S$. agalactiae, respectively. The inhibitory effect of the monoclonal antibody was not as high as the polyclonal serum, but $E$. coli 0157:H7 invasion was inhibited about $43 \%$ as compared with untreated $E$. coli $0157: \mathrm{H7}$, but not inhibited $15 \%$ as compared with polyclonal serum. The invasion of $K$. pneumoniae and S. agalactiae was not as high as polyclonal serum but about $8 \%$ to $10 \%$ was weaker than polyclonal serum.

Bacterial survival in the amoeba and the effect of MBP antibodies

The invaded bacteria were subsequently incubated for one hour for survival within amoeba cytoplasm. The surviving bacteria were calculated as mentioned at association and invasion assay (Fig. 3). Overall, survived bacteria were decreased as compared with invasion results, suggesting that some bacteria were killed within amoeba cytoplasm. Interestingly, the pathogenic strains of $K$. pneumoniae and S. agalactiae showed less decrease in survival as shown at invasion than $E$. coli 0157:H7 without antibody, suggesting that pathogenicity should be an important factor in survival. Moreover, the polyclonal and monoclonal antibody showed similar decreasing patterns.

\section{DISCUSSION}

Bacteria can interact and communicate with protozoan parasites, e.g., Acanthamoeba by receptor-ligand binding. Outer-membrane protein A (OmpA) and lipopolysaccharide (LPS) were important bacterial factors responsible for the interaction between $A$. castellanii and pathogenic $E$. coli $\mathrm{K} 1^{4}$. On the other hand, an interesting molecule associated with the contact-dependent pathway was MBP in A. castellanii which had an effect on the contact-dependent cytotoxicity to host cells ${ }^{12,22}$. It was clear that MBP in A. castellanii interacted with alpha-mannose of saccharides in bacteria. The monoclonal antibody to MBP in $A$.

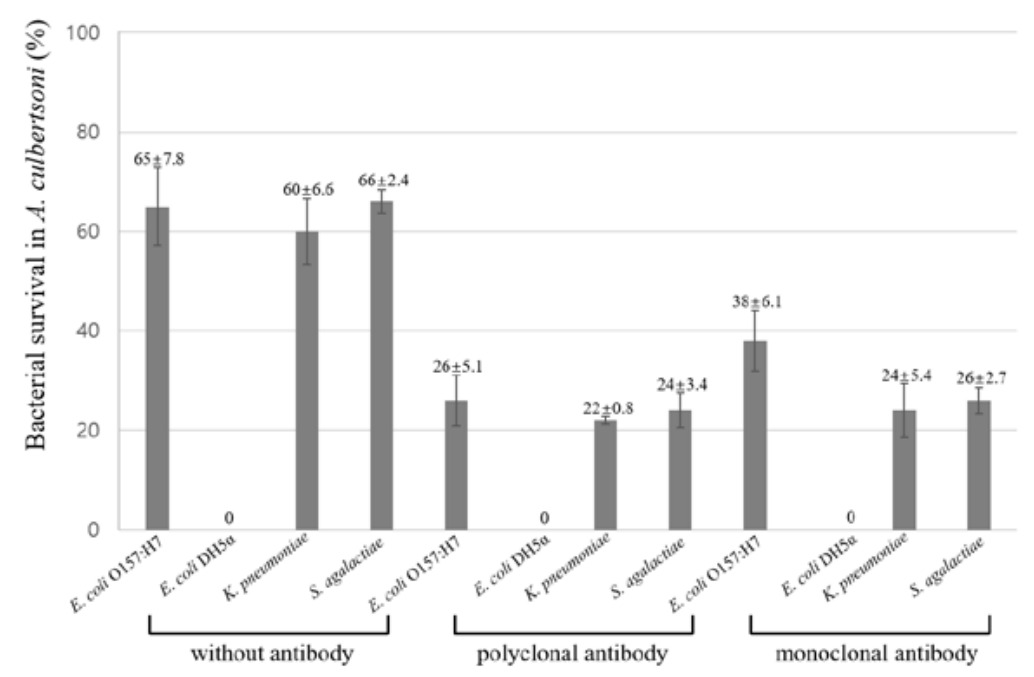

Fig. 3. Percentage of bacterial survival in A. culbertsoni trophozoites. Bacteria incubated with gentamicin were allowed to survive within the amoebae. Survival assay was done post to the invasion assay above. This experiment was performed in triplicate wells with three times, and data were described by mean \pm standard deviation (SD) value. 
culbertsoni, DG11 of IgM of kappa chains detected $83 \mathrm{kDa}$ on gels was produced ${ }^{13}$. It was applied to analyze the interaction between $A$. culbertsoni trophozoites and several bacteria in this study. The MBP had very important aspects because the MBP was expressed higher in the movement which could be done by pseudopodia, implying that the MBP could be one of the crucial factors in their interactions.

Three pathogenic bacteria such as E. coli 0157:H7, K. pnuemoniae, S. agalactiae and one non-pathogenic $E$. coli $\mathrm{DH} 5 \alpha$ showed different patterns of bacterial interactions with A. culbertsoni trophozoites and their interactions were inhibited by the pre-incubation of polyclonal serum and monoclonal antibody to MBP into $A$. culbertsoni. According to Alsam et al., 20064, the interaction between $E$. coli $\mathrm{K} 1$ and $A$. castellanii was regulated by the pathogenicity of $E$. coli. Similar patterns were observed in this study as pathogenic E. coli 0157:H7, K. pnuemoniae, S. agalactiae were higher in the interactions than a non-pathogenic strain. The size of bacteria varies around 0.5 to $2 \mu \mathrm{m}$ and the amoeba has the size of up to $25 \mu \mathrm{m}$. Pathogenic E. coli 0157:H7 and $K$. pnuemoniae are generally bigger than $S$. agalactiae even though $S$. agalactiae forms a long chain. Our results showed that there was not significant difference between rod type and long coccus type by size. Amoeba showed the result of bacteria predation through interaction with bacteria. However, like macrophages, the exact mechanism of bacterial predation within amoeba, such as autophagy, is not yet known, but protein-protein interactions will analyze similar autophagy functions in amoeba ${ }^{23}$. The results showed that pathogenic $E$. coli $0157: \mathrm{H} 7$ survived in amoeba by about $65 \%$. Since amoeba can eat and live with bacteria, bacteria can exist for a long time in amoeba. Bacteria and fungi are still present in women with abortions ${ }^{24}$. In particular, about $14 \%$ of Ureaplasma parvum was detected by PCR. Since macrophages have the ability to phagocytose bacteria, the interaction of bacteria, such as amoeba and $U$. parvum, used in this experiment instead of macrophages, may be helpful in studying bacterial survival. This study provided the information that the pathogenic bacteria could be more interactive with $A$. culbertsoni trophozoites as a reservoir host than non-pathogenic $E$. coli
$\mathrm{DH} 5 \alpha$, and the amoeba should interact with bacteria by the MBP lectin.

In this project by using pathogenic and non-pathogenic bacteria such as E. coli O157:H7, K. pnuemoniae, S. agalactiae and E. coli $\mathrm{DH} 5 \alpha$, it was analyzed whether a polyclonal serum and a monoclonal antibody to MBP of $A$. culbertsoni could inhibit its interaction such as bacterial association, invasion and survival. Moreover, it was analyzed that how much interaction was different when compared with interaction untreated with antiserum or antibody. We demonstrated that pathogenic bacteria could be more interactive with $A$. culbertsoni trophozoites as a reservoir host than non-pathogenic $E$. coli $\mathrm{DH} 5 \alpha$, and the amoeba should interact with bacteria by the MBP lectin.

\section{ACKNOWLEDGMENTS}

I would like to express our heartfelt thanks to Professor Naveed Khan for helping experimental procedures. I would like to thank Melissa Watkins at Namseoul University, South Korea for editing English as a native speaker.

\section{FUNDING}

Funding for this paper was provided by Namseoul university.

\section{ETHICS STATEMENT}

This article does not contain any studies with human participants or animals performed by any of the authors.

\section{DATA AVALIBILITY}

All datasets generated or analyzed during the study are included in the manuscript.

\section{REFERENCES}

1. Marciano-Cabral F, Cabral G. Acanthamoeba spp. as agents of disease in humans. Clin Microbiol Rev. 2003;16(2):273-307. doi: $10.1128 / \mathrm{cmr}$.16.2.273307.2003

2. Khan NA. Acanthamoeba: biology and increasing importance in human health. FEMS Microbiol Rev. 2006;30(4):564-595. doi: 10.1111/j.15746976.2006.00023.x

3. Jung S-Y, Matin A, Kim KS, Khan NA. The capsule plays an important role in Escherichia coli $\mathrm{K} 1$ interactions with Acanthamoeba. Int J Parasitol. 2007;37(3-4):417423. doi: 10.1016/j.ijpara.2006.10.012

4. Alsam S, Jeong SR, Sissons J, Dudley R, Kim KS, Khan NA. Escherichia coli interactions with Acanthamoeba: a symbiosis with environmental and clinical implications. 
J Med Microbiol. 2006;55(6):689-694. doi: 10.1099/ jmm.0.46497-0

5. Rowbotham TJ. Preliminary report on the pathogenicity of Legionella pneumophila for freshwater and soil amoebae. J Clin Pathol. 1980;33:1179-1183. doi: 10.1136/jcp.33.12.1179

6. Michel R, Burghardt H, Bergmann H. Acanthamoeba, naturally intracellularly infected with Pseudomonas aeruginosa, after their isolation from a microbiologically contaminated drinking water system in a hospital. Zentralbl Hyg Umweltmed. 1995;196(6):532-544.

7. Swart AL, Harrison CF, Eichinger L, Steinert M, Hilbi H. Acanthamoeba and Dictyostelium as Cellular Models for Legionella Infection. Front Cell Infect Microbiol. 2018;2:8-61. doi: 10.3389/fcimb.2018.00061

8. Hoffmann C, Finsel I, Hilbi H. Pathogen vacuole purification from Legionella-infected amoeba and macrophages. Methods Mol Biol. 2012;954:309-321. doi: 10.1007/978-1-62703-161-5_18

9. Bergmann $S$, Steinert M. From single cells to engineered and explanted tissues: new perspectives in bacterial infection biology. Int Rev Cell Mol Biol. 2015;319:1-44. doi: 10.1016/bs.ircmb.2015.06.003

10. Gao S, Lewis GD, Ashokkumar M, Hemar Y. Inactivation of microorganisms by low-frequency high-power ultrasound: 1. Effect of growth phase and capsule properties of the bacteria. Ultrason Sonochem. 2014;21(1):446-453. doi: 10.1016/j. ultsonch.2013.06.006

11. Rizzo J, Albuquerque PC, Wolf JM, et al. Analysis of multiple components involved in the interaction between Cryptococcus neoformans and Acanthamoeba castellanii. Fungal Biol. 2017;121(6-7):602-614. doi: 10.1016/j.funbio.2017.04.002

12. Kim JH, Matin A, Shin HJ, et al. Functional roles of mannose-binding protein in the adhesion, cytotoxicity and phagocytosis of Acanthamoeba castellanii. Exp Parasitol. 2012;132(2):287-292. doi: 10.1016/j. exppara.2012.08.007

13. Kang A-Y, Park A-Y, Shin H-J, Khan NA, Maciver SK, Jung SY. Production of a monoclonal antibody against a mannose-binding protein of Acanthamoeba culbertsoni and its localization. Exp Parasitol. 2018;192:19-24. doi: 10.1016/j.exppara.2018.07.009
14. Kim YK, Kim TU, Joung IS, Im KI. A comparative study on hydrolase activities in Acanthamoeba culbertsoni and A. royreba. Kor J Parasitol. 1988;26(2):95-106. doi: 10.3347/kjp.1988.26.2.95

15. Kong $\mathrm{HH}$, Seo SA, Shin CO, Im KI. The effect of immunization with Acanthamoeba culbertsoni in mice born to immune mother. Kor J Parasitol. 1993;31(2):157-163. doi: 10.3347/kjp.1993.31.2.157

16. Krishna-Prasad BN, Gupta SK. Preliminary report on the engulfment and retention of Mycobacteria by trophozoites of axenically grown Acanthamoeba castellanii Douglas. Curr Sci. 1978;47:245-247.

17. Yoo KT, Jung SY. Effects of mannose on pathogenesis of Acanthamoeba castellanii. Kor J Parasitol. 2012;50(4):365-369. doi: 10.3347/kjp.2012.50.4.365

18. Lim KB, Boey LP, Khatijah M. Gram's-stained microscopy in the etiological diagnosis of Malassezia (Pityrosporon) folliculitis. Arch Dermatol. 1988;124(4):492.

19. Garate M, Cubillos I, Marchant J, Panjwani N. Biochemical characterization and functional studies of Acanthamoeba mannose-binding protein. Infect Immun. 2005;73(9):5775-5781. doi: 10.1128/ IAI.73.9.5775-5781.2005

20. Kim J-H, Lee J, Sohn H-J, et al. Production of monoclonal antibodies for Plasmodium vivax lactate dehydrogenase and patient sera screening using sandwich ELISA. Parasitol Res. 2012;111(4):1645-1650. doi: 10.1007/s00436-012-3003-x

21. Song KJ, Jung SY. Biocidal effects of chlorine dioxide on isolated and identified pathogens from nosocomial environment-biochemical and technical convergence. J Digit Converg. 2017;15(6):339-344.

22. Garate M, Cao Z, Bateman E, Panjwani N. Cloning and characterization of a novel mannose-binding protein of Acanthamoeba. J Biol Chem. 2004;279(28):2984929905. doi: $10.1074 / \mathrm{jbc}$ M402334200

23. Bah A, Vergne I. Macrophage autophagy and bacterial infections. Front Immunol. 2017;8:1483. doi: 10.3389/ fimmu.2017.01483

24. Contini C, Rotondo JC, Magagnoli F, et al. Investigation on silent bacterial infections in specimens from pregnant women affected by spontaneous miscarriage. J Cell Physiol. 2018;234(1):100-107. doi: 10.1002/ jcp.26952. 